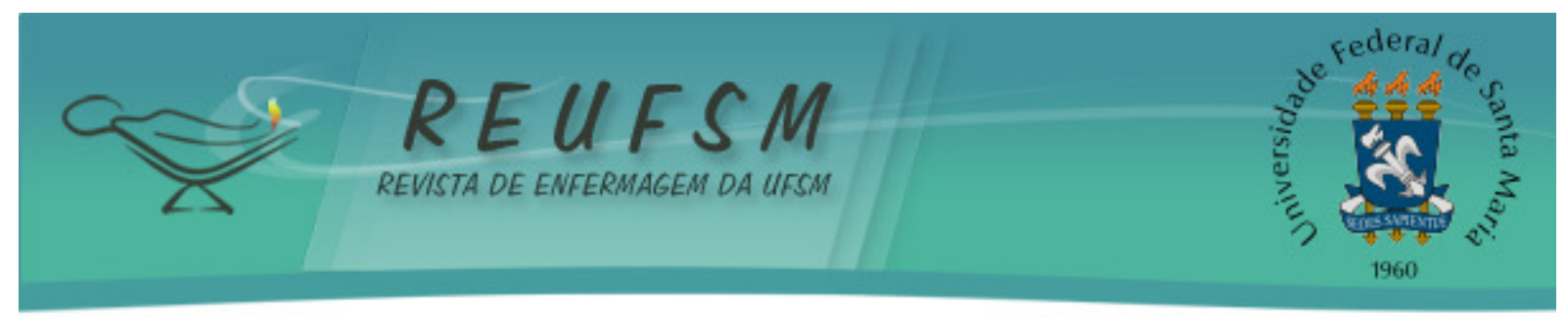

ARTIGO ORIGINAL

\title{
NÍVEL DE COMPLEXIDADE ASSISTENCIAL DE PACIENTES E O QUANTITATIVO DE PROFISSIONAIS DE ENFERMAGEM
}

\section{COMPLEXITY LEVEL OF PATIENT ASSESSMENT AND THE AMOUNT OF PROFESSIONAL NURSING}

\section{NIVEL DE COMPLEJIDAD DE LA ATENCIÓN AL PACIENTE Y CUANTITATIVO DE ENFERMERÍA PROFESIONAL}

\author{
Halana Batistel Barbosa ${ }^{1}$ \\ Lara Adrianne Garcia Paiano² \\ Anair Lazzari Nicola ${ }^{3}$ \\ Luciana Magnani Fernandes ${ }^{4}$
}

Doi: $10.5902 / 217976929230$

RESUMO: Objetivo: identificar o quantitativo de profissionais de enfermagem para atender as necessidades de assistência dos pacientes frente ao seu nível de complexidade assistencial nas unidades de Clínica Médica e Cirúrgica e, Neurologia e Ortopedia de um hospital universitário. Método: estudo descritivo com abordagem quantitativa. Resultados: identificou-se uma média de 5,5 e 11,7 pacientes em cuidado semi-intensivo e, 9,9 e 6,1 pacientes em cuidado intensivo nas Unidades I e II respectivamente, com indicação de internação em Unidade de Terapia Intensiva que, no entanto são atendidos em unidades de internamento comuns, que, geralmente não possuem infraestrutura física e material adequada para assistir essa clientela. Conclusão: a proporção do quantitativo de profissionais é inadequada para prestar assistência com segurança e qualidade, apontando a necessidade de reorganização do processo de trabalho referente ao agrupamento de pacientes por complexidade assistencial.

Descritores: Enfermagem; Recursos humanos; Classificação.

ABSTRACT: Aim: to identify the number of nurse professionals to meet the care needs of patients against their level of assessment complexity in units of Medical and Surgical Clinic and, Neurology and Orthopedics of a university hospital. Method: Descriptive study with a quantitative approach.Results: identified an average of 5.5 and 11.7 patients in semiintensive care, and 9.9 and 6.1 in patients in intensive care in the Units I and II respectively, indicating hospitalization in Intensive Care Unit that are treated in internment units common, however generally do not have adequate physical infrastructure and equipment to assist these clients. Conclusion: The proportion of quantitative of professionals is inadequate to assist with safety and quality, suggesting the need of reorganization of working process relative to the grouping of patients for assistance complexity.

Descriptors: Nursing; Human resources; Classification.

\footnotetext{
${ }^{1}$ Enfermeira Residente do Programa de Residência em Enfermagem na especialidade Gerenciamento de Enfermagem em Clínica Médica e Cirúrgica da Universidade Estadual do Oeste do Paraná - UNIOESTE, Campus Cascavel, PR, Brasil. Email: halanabarbosa@hotmail.com.

${ }^{2}$ Enfermeira Residente do Programa de Residência em Enfermagem na especialidade Gerenciamento de Enfermagem em Clínica Médica e Cirúrgica da Universidade Estadual do Oeste do Paraná - UNIOESTE, Campus Cascavel, PR, Brasil. Email: laraagp@hotmail.com.

${ }^{3}$ Enfermeira Doutora em Enfermagem, Docente do curso de Enfermagem da Universidade Estadual do Oeste do Paraná - UNIOESTE, Campus Cascavel, PR, Brasil. Email: anairln@yahoo.com.br.

${ }^{4}$ Enfermeira Doutora em Enfermagem, Docente do curso de Enfermagem da Universidade Estadual do Oeste do Paraná - UNIOESTE, Campus Cascavel, PR, Brasil. Email: lumagna@terra.com.br.
} 


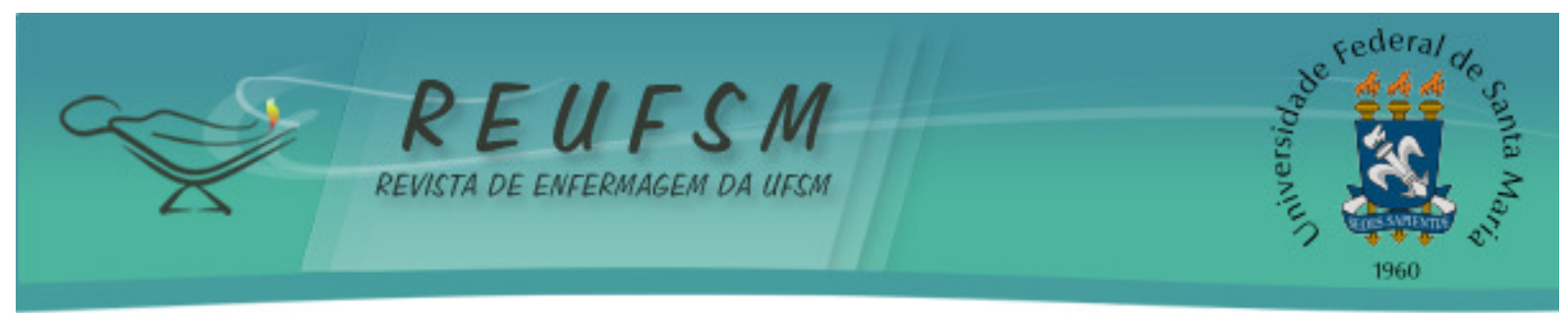

RESUMEN: Objetivo: identificar el cuantitativo de enfermeros para satisfacer las necesidades de atención de los pacientes relativo al nivel de complejidad de atención en las unidades de Clínica Médica y Quirúrgica, Neurología y Ortopedia de un hospital universitario. Método: estudio descriptivo con enfoque cuantitativo. Resultados: Fue identificado una media de 5.5 y 11.7 pacientes en cuidado semiintensivo, y 9.9 y 6.1 pacientes en cuidado intensivo en las Unidades I y II respectivamente, con indicación de internación en la Unidad de Cuidados Intensivos, todavía estos pacientes son atendidos en las enfermerías comunes, que generalmente no tienen una infraestructura física y material adecuados para asistir a estos clientes. Conclusión: la proporción del cuantitativo de los profesionales es insuficiente para asistir con seguridad y calidad esos pacientes, señalando la necesidad de reorganizar el proceso de trabajo relativo al grupo de pacientes por complejidad asistencial.

Descriptores: Enfermería; Recursos humanos; Clasificación.

\section{INTRODUÇÃO}

Nas instituições hospitalares, os profissionais de enfermagem representam um papel fundamental no processo assistencial e, por isso, constitui-se numa parcela significativa de seu quadro de pessoal. Os aspectos quantitativos de profissionais de enfermagem nessas instituições são enfatizados para que haja a garantia da segurança e da qualidade da assistência ao paciente. ${ }^{1}$

A qualidade da assistência está diretamente relacionada com os recursos disponíveis e como eles são utilizados na prática. Entre os recursos que interferem nos resultados da assistência está o quantitativo de profissionais de enfermagem que pode representar até $60 \%$ da força de trabalho nas instituições hospitalares. ${ }^{1-2}$

Diante desse contexto, os enfermeiros devem utilizar instrumentos que auxiliem no planejamento, distribuição e controle adequado dos profissionais de enfermagem, bem como identificar as variáveis que interferem na carga de trabalho da equipe de enfermagem. ${ }^{1-3}$ Nas instituições hospitalares, tem-se procurado cada vez mais adotar estratégias que garantam a qualidade da assistência com menor custo, visando o cumprimento das ações básicas de saúde e o atendimento da alta regulamentação do setor, visto que, os serviços prestados devem ir ao encontro das necessidades dos usuários. ${ }^{3-4}$ Enfatizando as condições das instituições hospitalares públicas atuais, sabe-se que as categorias profissionais da enfermagem vêm sofrendo com a sobrecarga de trabalho devido à incoerência entre quantitativo de profissionais e demanda assistencial. ${ }^{5}$

A operacionalização do dimensionamento de profissionais de enfermagem pela aplicação de um método possibilita interelacionar e mensurar as variáveis que interferem na carga de trabalho da equipe de enfermagem. A variável carga de trabalho é mensurada a partir da aplicação do um Sistema de Classificação de Paciente (SCP), que determina o grau de dependência de cada paciente e o tempo dispendido no cuidado direto e indireto para atender as necessidades de assistência. ${ }^{1-2}$

Em consonância com a Resolução n 293/2004 do Conselho Federal de Enfermagem (COFEn), o dimensionamento do quadro de profissionais de enfermagem deve basear-se em características relativas à instituição, ao serviço de enfermagem e à clientela. Tal resolução estabelece parâmetros para o dimensionamento de pessoal de enfermagem nas instituições de saúde, com base na aplicação de um Sistema de Classificação de Pacientes, como critério para se estabelecer o perfil de cada paciente internado, as horas mínimas de assistência e a distribuição dos profissionais para cada tipo de cuidado. ${ }^{6}$

Nessa perspectiva, o presente estudo desenvolveu-se a partir da questão norteadora: "o quantitativo de profissionais de enfermagem é suficiente para atender as necessidades de assistência dos pacientes em unidades de internamento?" Para tanto, definiu-se como objetivo identificar o quantitativo de profissionais de enfermagem para 


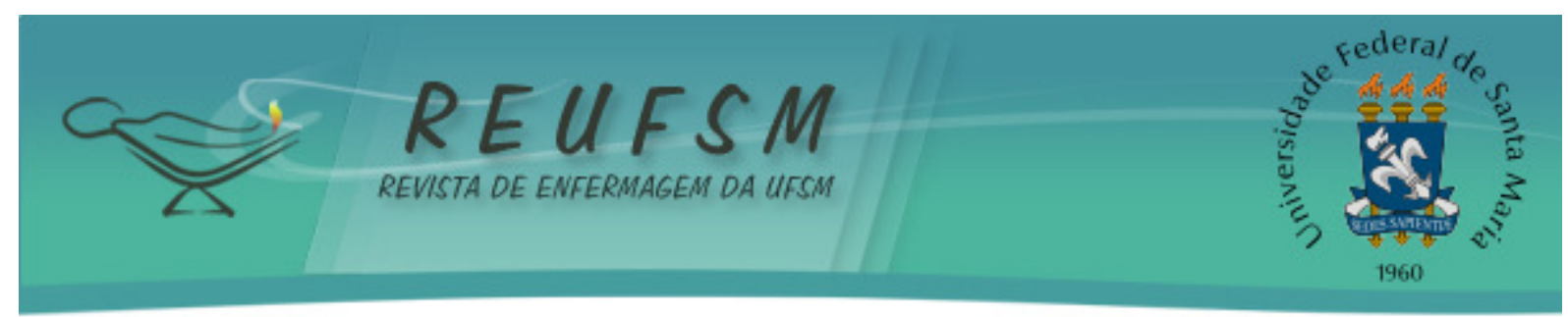

atender as necessidades de assistência dos pacientes frente ao seu nível de complexidade assistencial nas unidades de Clínica Médica e Cirúrgica e, Neurologia e Ortopedia de um hospital universitário.

\section{MÉTODO}

Estudo descritivo com abordagem quantitativa realizado nas unidades de Clínica Médica e Cirúrgica (Unidade I) e Neurologia e Ortopedia (Unidade II) de um hospital público do Oeste do Paraná, com capacidade para 28 e 26 leitos respectivamente, dos quais, na Unidade I, 13 leitos eram destinados aos pacientes clínicos e 15 aos pacientes cirúrgicos e, na Unidade II, dez eram destinados aos pacientes da ortopedia e 16 aos pacientes neurológicos.

Utilizou-se para identificar o nível de complexidade assistencial, o Sistema de Classificação de Pacientes (SCP) que, avalia o paciente de acordo com o grau de dependência nas seguintes áreas de cuidado: estado mental; oxigenação; sinais vitais; motilidade; deambulação; alimentação; cuidado corporal; eliminação; terapêutica; integridade cutâneo-mucosa/comprometimento tecidual; curativo e tempo utilizado na realização de curativos. Partindo da pontuação obtida nessa avaliação, é possível classificar os pacientes em categorias de cuidado, quais sejam: cuidado intensivo (acima de 34 pontos), cuidado semi-intensivo (29-34 pontos), cuidado alta dependência (23-28 pontos), cuidado intermediário (18-22 pontos) e cuidado mínimo (12-17 pontos). ${ }^{5}$

O SCP adotado define estas cinco áreas de cuidado, entretanto, a Resolução № 293/2004 do Conselho Federal de Enfermagem (COFEn), classifica a complexidade do cuidado em quatro categorias: cuidados intensivos, semi-intensivos, intermediários e mínimos. Para fins deste estudo, na análise condensou-se os dados de alta dependência com os cuidados semi-intensivos. ${ }^{1-6}$

Para avaliar a carga de trabalho e para determinar o número de horas de assistência de enfermagem, utilizou-se a Resolução N 293/2004 do Conselho Federal de Enfermagem (COFEn) que, fixa e estabelece os parâmetros para o dimensionamento do quadro de profissionais de enfermagem nas unidades assistenciais de saúde ${ }^{1-6}$

A amostra do estudo foi composta de 125 prontuários eletrônicos na Unidade I, no período de primeiro a 30 de julho do ano de 2012 e, 114 prontuários eletrônicos de pacientes internados na Unidade II, no período de primeiro a 28 de julho de 2012. 0 período foi definido de acordo com a permanência dos enfermeiros residentes nas unidades, sendo incluídos na amostragem todos os pacientes internados, não havendo critérios de exclusão. O SCP compõe o prontuário eletrônico do paciente e era preenchido, diariamente, pelos enfermeiros das unidades e enfermeiros residentes.

Tal sistema prevê o agrupamento de pacientes em consonância com seu nível de complexidade assistencial, contribuindo para a realização de um cuidado individualizado para grupos de pacientes com necessidades específicas visando, dessa forma, maior efetividade do cuidado. ${ }^{7-8}$

Para identificar o número de profissionais que prestavam serviço no período de coleta de dados nas duas unidades, utilizou-se a escala mensal de enfermagem adotada na instituição, na qual foram avaliados todos os turnos de trabalho. A equipe de trabalho desses setores era composta por um enfermeiro por turno de trabalho e uma média de seis auxiliares/técnicos de enfermagem também por turno de trabalho.

O cálculo das horas de enfermagem por tipo de cuidado foi realizado mediante disposições da referida resolução, a qual determina que, o referencial mínimo para o quadro de profissionais de enfermagem para as 24 horas de cada unidade de internação, considera o nível de complexidade do paciente, definido pelo SCP.

Nesse sentido, considera-se como horas de enfermagem/leito nas 24 horas: 3,8 horas de enfermagem por cliente, na assistência mínima; 5,6 horas de enfermagem por 


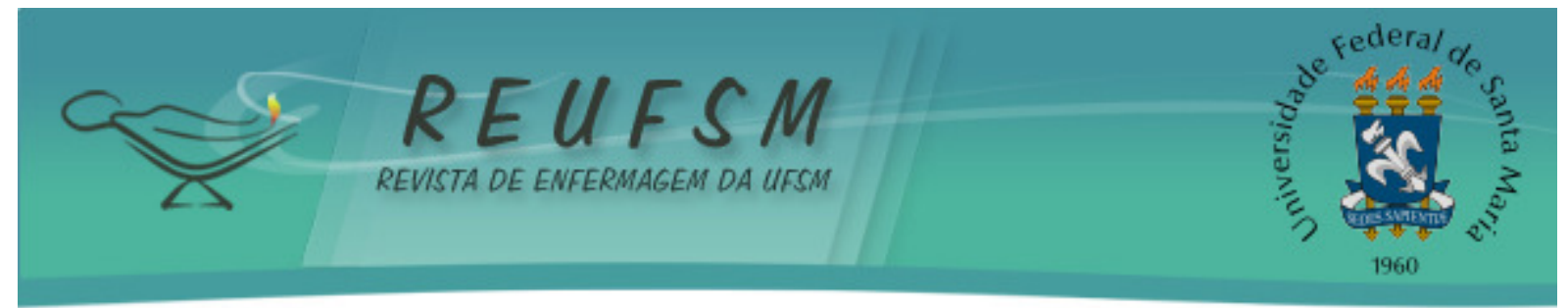

cliente na assistência intermediária; 9,4 horas de enfermagem por cliente na assistência semi-intensiva e 17,9 horas de enfermagem por cliente na assistência intensiva. ${ }^{6}$

0 cálculo do pessoal de enfermagem segundo o tempo médio diário de cuidado preconizado pela resolução do COFEn, permite que sejam levantados dados referentes à carga média diária de trabalho, participação no tempo de cuidado (percentagem de horas necessárias para cada categoria de cuidado), carga média diária de trabalho por categoria profissional, total da carga diária de trabalho, a estimativa do quantitativo de profissionais necessários à realização do cuidado. ${ }^{6}$ Os dados foram analisados por meio da estatística descritiva, com auxílio do Programa Microsoft Excel 2010® e apresentado na forma de tabelas.

O projeto de pesquisa foi aprovado pelo Comitê de Ética da Universidade Estadual do Oeste do Paraná sob o registro 014/2011, atendendo à Resolução 196/96 do Conselho Nacional de Saúde.

\section{RESULTADOS E DISCUSSÃO}

$\mathrm{Na}$ Unidade I, 59 (47,3\%) pacientes eram do sexo feminino e $66(52,8 \%)$ do sexo masculino. Em relação à idade, $92(73,6 \%)$ eram adultos e $33(26,4 \%)$ eram idosos, sendo considerados idosos pacientes com idade igual ou maior que 60 anos. Do total de 125 pacientes, 45 (36\%) permaneceram com acompanhante devido ao quadro clínico, dos quais $27(81,8 \%)$ eram idosos, e $80(64 \%)$ pacientes não permaneceram com acompanhante.

Conforme os dados apresentados (Tabela 1 ) evidenciou-se que a carga média de trabalho gerada pelos pacientes classificados como semi-intensivo e intensivo geraram maior carga horária para o profissional enfermeiro. Nesse sentido, compete ao enfermeiro dimensionar e gerenciar o quadro de profissionais de enfermagem visto que, a Unidade I atende diariamente pacientes de todas as categorias de cuidado.

Tabela 1- Distribuição média de pacientes por complexidade assistencial e carga média diária de trabalho necessária por categoria profissional da Unidade I. Hospital Universitário do Oeste do Paraná. Cascavel/PR, Brasil. 2012.

\begin{tabular}{|c|c|c|c|c|c|}
\hline Nível de complexidade & IT & SI & ITM & $M$ & Total \\
\hline Média de pacientes & 1,8 & 5,5 & 9,9 & 6,4 & 23,6 \\
\hline $\begin{array}{lll}\text { Carga média de } \\
\text { trabalho }\end{array}$ & 32,2 & 51,7 & 55,4 & 24,3 & 163,7 \\
\hline $\begin{array}{l}\text { Carga média necessária } \\
\text { do enfermeiro }\end{array}$ & 18,0 & 23,8 & 20,5 & 9,0 & 71,3 \\
\hline $\begin{array}{l}\text { Carga média necessária } \\
\text { do aux/tec }\end{array}$ & 14,2 & 27,9 & 34,9 & 15,3 & 92,3 \\
\hline
\end{tabular}

Nota: IT refere-se a pacientes intensivos; SI a pacientes semi-intensivos; ITM a pacientes intermediários e $\mathrm{M}$ a pacientes de cuidado mínimo.

No que se refere à distribuição das horas de assistência entre as categorias profissionais na Unidade I, procedeu-se conforme indicação da Resolução, evidenciando uma carga média de trabalho de 163,7 horas/dia, sendo 71,3 horas/dia desse total correspondente ao tempo de trabalho do enfermeiro e, 92,3 horas/dia ao trabalho do auxiliar/técnico de enfermagem, considerando a média de 23,6 pacientes/dia.

A identificação da carga de trabalho e a mensuração do tempo que a enfermagem utiliza para prestar a assistência tanto direta quanto indireta aos pacientes são fatores que possibilitam determinar e avaliar quantitativa e qualitativamente os profissionais de enfermagem. ${ }^{1-4}$

Os pacientes considerados como de cuidados mínimos caracterizam-se por serem pacientes pré-operatórios de cirurgias eletivas de pequeno, médio e grande porte, que evoluem para a classificação de cuidados intermediários e de cuidados semi-intensivos no 


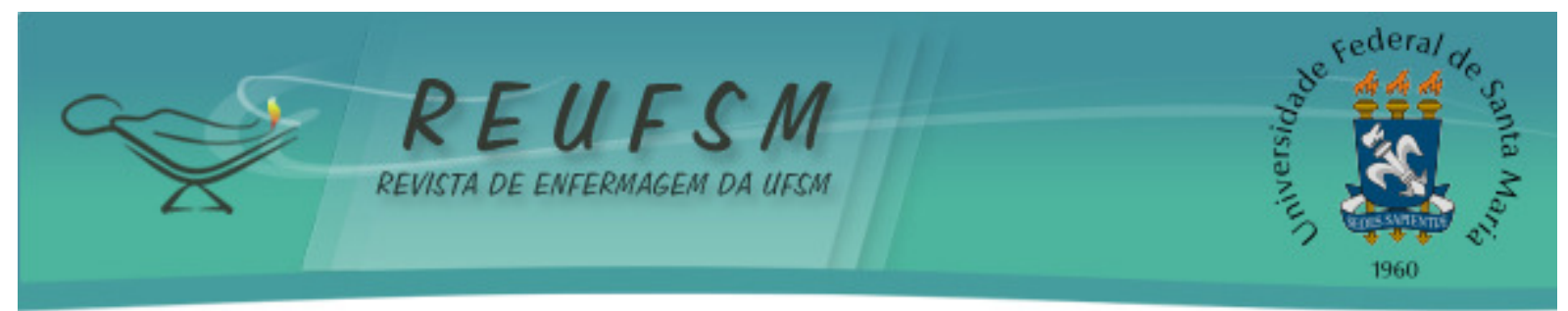

pós-operatório imediato e mediato em razão das limitações físicas resultantes do procedimento cirúrgico.

No pós-operatório imediato uma justificativa para a progressão da classificação de cuidados dos pacientes é que o hospital em questão não possui sala de recuperação pós-anestésica adequada no centro cirúrgico. Dessa forma, os pacientes retornam à unidade ainda sob efeito anestésico e requerem maior vigilância da equipe de enfermagem devido ao seu grau de dependência.

No pós-operatório mediato, a classificação de cuidados dos pacientes evolui devido à necessidade de cuidados de enfermagem específicos como: curativos, sondagens, dispositivos para dietas, além da possibilidade de ocorrência de complicações pós-operatórias.

Conforme evidenciou-se, os dados elencados na Tabela 02, a Unidade I também assiste pacientes que internam para tratamentos clínicos com maior tempo de internamento que os pacientes cirúrgicos, demandando grande carga de trabalho da equipe de enfermagem, pois são em sua maioria, pacientes idosos, acamados, incapazes de movimentar qualquer segmento corporal. Portanto, com necessidade de mudança de decúbito e movimentação passiva programada e realizada pela enfermagem, que apresentavam dificuldade para movimentar-se ou alguma limitação de movimentos, com necessidade de banho no leito realizado pela enfermagem. ${ }^{9}$

Tabela 2: Itens do Sistema de Classificação de Pacientes que obtiveram maiores pontuações na Unidade I. Hospital Universitário do Oeste do Paraná. Cascavel/PR, Brasil. 2012.

\begin{tabular}{lcc}
\hline Item com maior pontuação no SCP & Número de pacientes & $\%$ \\
Uso contínuo de mascara ou cateter & 40 & 32,0 \\
de oxigênio & & \\
$\begin{array}{l}\text { Ventilação mecânica (uso de } \\
\text { ventilador a pressão ou a volume) }\end{array}$ & 34 & 27,2 \\
$\begin{array}{l}\text { Limitação de movimentos } \\
\text { Dificuldade para movimentar }\end{array}$ & 80 & 64,0 \\
$\begin{array}{l}\text { seguimentos corporais, mudança de } \\
\text { decúbito, movimentação passiva }\end{array}$ & 53 & 42,5 \\
auxiliada pela enfermagem de movimentar qualquer & & \\
$\begin{array}{l}\text { Incapaz de } \\
\text { seguimento corporal, mudança de } \\
\text { decúbito e movimentação passiva } \\
\text { programada e realizada pela } \\
\text { enfermagem }\end{array}$ & 42 & 33,6 \\
Banho no leito, higiene oral \\
realizada pela enfermagem
\end{tabular}

Outrossim, na unidade encontravam-se pacientes traqueostomizados, submetidos a oxigenoterapia contínua, sendo, muitas vezes, necessário o uso de ventilação mecânica, ou seja, eram pacientes altamente dependentes dos cuidados da enfermagem.

No que tange à caracterização dos pacientes da Unidade II, $69(60,5 \%)$ eram do sexo feminino e $45(39,5)$ eram do sexo masculino. Em relação à idade, $81(71 \%)$ eram adultos e $33(29 \%)$ eram idosos. Dos 81 pacientes adultos, 18 (22,2\%) permaneceram com acompanhante devido ao quadro clínico de alta dependência, os $63(77,8 \%)$ pacientes restantes permaneceram sem. No entanto dos 33 pacientes idosos, $27 \quad(81,8 \%)$ permaneceram com acompanhantes e seis $(18,2 \%)$ sem.

A Tabela 3 demonstra a expressiva carga média de trabalho gerada pelos pacientes classificados como semi-intensivos, o que implica em maior carga média de 


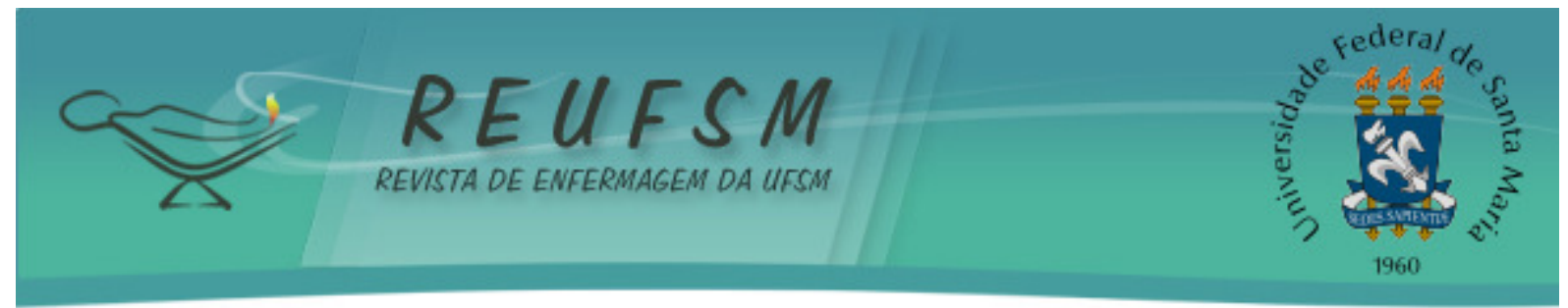

trabalho para o enfermeiro, corroborando com o encontrado na Unidade I, o que se deve às características da unidade.

Tabela 3 - Distribuição média de pacientes por complexidade assistencial e carga média diária de trabalho necessária por categoria profissional da Unidade II. Hospital Universitário do Oeste do Paraná. Cascavel/PR, Brasil. 2012.

\begin{tabular}{|c|c|c|c|c|c|}
\hline \multirow{2}{*}{$\begin{array}{l}\text { Nível de complexidade } \\
\text { Média de pacientes }\end{array}$} & IT & $\mathrm{SI}$ & ITM & $M$ & Total \\
\hline & 1,3 & 11,7 & 6,1 & 1,8 & 20,9 \\
\hline $\begin{array}{l}\text { Carga média de } \\
\text { trabalho }\end{array}$ & 23,3 & 110,0 & 46 & 50,6 & 174,5 \\
\hline $\begin{array}{l}\text { Carga média necessária } \\
\text { do enfermeiro }\end{array}$ & 13,0 & 50,6 & 12,6 & 2,5 & 78,8 \\
\hline $\begin{array}{l}\text { Carga média necessária } \\
\text { do aux/tec }\end{array}$ & 10,2 & 59,4 & 21,5 & 4,3 & 95,5 \\
\hline
\end{tabular}

Nota: IT refere-se a pacientes intensivos; SI a pacientes semi-intensivos; ITM a pacientes intermediários e $\mathrm{M}$ a pacientes de cuidado mínimo.

No que se refere à distribuição das horas de assistência entre as categorias profissionais na Unidade II, procedeu-se conforme indicação da Resolução, evidenciando uma carga média de trabalho de 174,5 horas/dia, sendo 78,8 horas/dia desse total correspondente ao tempo de trabalho do enfermeiro e, 95,5 horas/dia ao trabalho do auxiliar/técnico de enfermagem considerando a média de 20,9 pacientes/dia.

A Unidade II se caracteriza pela internação de pacientes vítimas de acidentes automobilísticos em decorrência de acidentes de trânsito, politraumatizados, pacientes com traumatismo crânio-encefálico (TCE), cirurgias ortopédicas eletivas, pacientes portadores de tumores cerebrais ou acidente vascular cerebral, tornando-os altamente dependentes para as atividades diárias.

Um aspecto relevante observado durante a coleta dos dados (Tabela 4) ao comparar as duas unidades no que tange ao nível de complexidade, foi a questão relacionada à avaliação dos curativos, visto que, o SCP apresentava lacunas no que diz respeito à classificação e avaliação de lesões que interferem e até determinam a assistência. 0 problema relacionado com o cuidado com as feridas consiste em preocupação por parte dos profissionais com os cuidados a serem ministrados nesses casos, haja vista que, os enfermeiros são os profissionais envolvidos diretamente na assistência a pacientes portadores de feridas. ${ }^{5}$

Tabela 4 - Itens do Sistema de Classificação de Pacientes que obtiveram maiores pontuações na Unidade II. Hospital Universitário do Oeste do Paraná. Cascavel/PR, Brasil. 2012.

\begin{tabular}{lcc}
\hline Item com maior pontuação no SCP & Número de pacientes & $\%$ \\
$\begin{array}{l}\text { Limitação de movimentos } \\
\text { Restrito ao leito }\end{array}$ & 268 & 45,9 \\
$\begin{array}{l}\text { Banho no leito, higiene oral } \\
\text { realizada pela enfermagem }\end{array}$ & 289 & 49,5 \\
$\begin{array}{l}\text { Curativo realizado uma vez ao dia } \\
\text { pela equipe de enfermagem }\end{array}$ & 414 & 49,1 \\
Entre 5 e 15 minutos & 370 & 70,9 \\
\hline
\end{tabular}

Embora o tempo destinado a execução dos curativos na Unidade II tenha apresentado maior pontuação no item "entre 5 e 15 minutos", muitos dos procedimentos realizados devido a característica clínica demandaram um tempo maior, exigindo do enfermeiro maior tempo de cuidado. A execução de extensos curativos pelo enfermeiro 


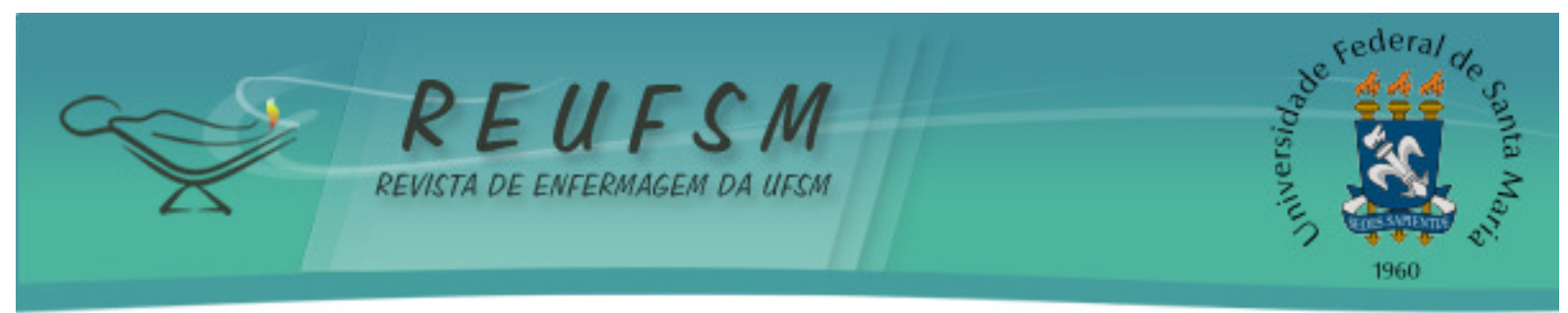

pode demandar desse profissional um tempo de assistência direta não compatível com o tempo estimado pelo SCP na realização de curativos, o que acaba por interferir no planejamento do quantitativo de profissionais necessários para atender as necessidades dos pacientes daquela unidade.

A primeira etapa do processo de dimensionamento de pessoal diz respeito à classificação da clientela internada sob o ponto de vista de necessidades de cuidado de enfermagem. Utilizando o SCP procedemos diariamente à classificação dos pacientes internados nas Unidades I e II segundo o nível de complexidade assistencial do instrumento. ${ }^{7}$

Cabe ressaltar que nas duas unidades é constante a presença de familiares junto aos pacientes de cuidados de alta dependência, semi intensivos e intensivos além dos pacientes idosos e isso, tem influenciado no tempo de assistência e na organização do trabalho de enfermagem, pois, observou-se que, mesmo não dispondo de preparo adequado para prestar o cuidado, os acompanhantes tem assumido muitas funções específicas da enfermagem como procedimentos relacionados à higiene, conforto e alimentação. No entanto, compete a equipe de enfermagem supervisionar esses cuidados bem como o desenvolvimento de práticas educativas afim de prevenir possíveis riscos e danos aos pacientes.

Em relação ao quantitativo de profissionais de enfermagem na Unidade I, os dados mostram que a mesma, conta com uma média de 2,78 enfermeiros/dia e 14,1 técnicos de enfermagem/dia. Dessa maneira, considerando a soma da carga média diária de trabalho gerada pelos pacientes (163,7 horas), segundo as recomendações da Resolução do Cofen, seria necessário a distribuição dos profissionais seguindo uma média diária de quatro enfermeiros e cinco auxiliares e técnicos de enfermagem por turno, totalizando 19,6 enfermeiros e 25,2 profissionais de nível médio, nos diferentes turnos para atender a demanda de assistência desses pacientes.

No que tange ao quantitativo de profissionais de enfermagem da Unidade II, os resultados mostraram uma média de 2,71 enfermeiros/dia e 16,7 técnicos de enfermagem/dia. Considerando a soma da carga média diária de trabalho gerada pelos pacientes (174,5 horas), segundo as recomendações da Resolução do Cofen, seria necessário a distribuição dos profissionais seguindo uma média de 4,4 enfermeiros e 5,2 auxiliares/técnicos de enfermagem por turno, totalizando 21,9 profissionais enfermeiros e 26,2 auxiliares/técnicos para atender a demanda desses pacientes.

$\mathrm{Na}$ instituição onde o estudo foi desenvolvido, os pacientes que permanecem nas unidades têm apresentado necessidades cada vez mais especializadas e complexas, o que imprime sobrecarga de trabalho à equipe de enfermagem, influencia e dificulta a implementação de medidas que favoreçam a qualidade da assistência prestada. ${ }^{10-11}$

Em ambas as unidades, os resultados apontaram para um quantitativo expressivo de pacientes que exigiram cuidados semi-intensivo e intensivos evidenciando a presença de pacientes graves e instáveis hemodinamicamente, com indicação compulsória de internação em Unidade de Terapia Intensiva (UTI), que necessitam de assistência médica e de enfermagem contínua e especializada.

Em consonância com a Resolução do COFEn, ${ }^{6}$ a carga horária gerada pelos pacientes de cuidados intensivos e internados na Unidade I, é de 31,5 horas diárias, das quais, 17,6 horas (56\%) da assistência deveriam ser executadas por enfermeiros e 13,9 (44\%) por auxiliares ou técnicos de enfermagem. Já a Unidade II a carga horária gerada pelos pacientes de cuidados intensivos é de 23,3 horas diárias, das quais 13 horas (56\%) da assistência deveriam ser executadas por enfermeiros e 10,2 (44\%) por auxiliares ou técnicos de enfermagem.

A problemática que se instala quando se tem a necessidade de assistir pacientes de cuidados intensivos em unidades de internamento consiste em que estas muitas vezes não possuem recursos físicos, materiais e nem recursos humanos qualificados, justamente por não serem unidades de terapia intensiva e pelo número de pacientes ali assistidos. 


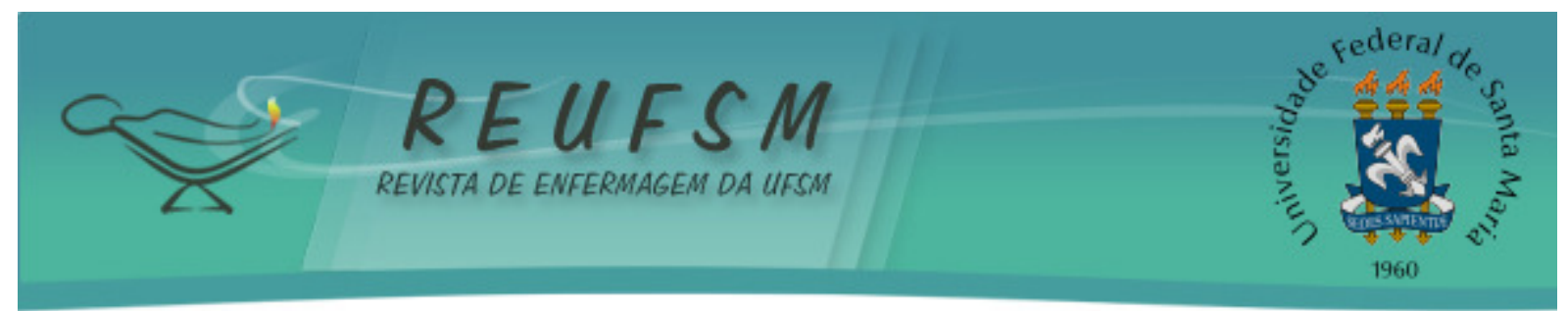

No cotidiano da prática profissional, o trabalhador de enfermagem se vê obrigado a prestar assistência a pacientes graves e dependentes fora de unidades de cuidados intensivos, em razão do número de leitos em UTI ser insuficiente para a demanda instalada. Estas unidades de internação geralmente não possuem o aparato e a infraestrutura de uma unidade de terapia intensiva e, muitas vezes, o trabalhador não está tecnicamente preparado para prestar cuidados com segurança a esse perfil de paciente, 0 que resulta em sobrecarga de trabalho e estresse ocupacional, que podem trazer consequências à sua saúde, além de prejuízos à instituição e ao paciente. ${ }^{10-11}$

Dessa forma, pelo sistema de classificação de pacientes, é possibilitado ao enfermeiro visualizar e caracterizar os pacientes assistidos nas unidades e o tipo de cuidado que deve ser prestado de acordo com o nível de complexidade, tornando imperativo que os enfermeiros tenham ampla formação e estejam habilitados para lidar com todos os tipos de pacientes. ${ }^{12}$

\section{CONCLUSÃO}

A aplicação do sistema de classificação de pacientes possibilitou a análise dos dados relacionados ao dimensionamento de pessoal nas unidades estudadas, evidenciando a inadequada proporção do quantitativo de profissionais para prestar assistência com segurança e qualidade aos pacientes. A análise das variáveis no processo de dimensionamento do pessoal de enfermagem apontou a necessidade de reorganização do processo de trabalho referente ao agrupamento de pacientes por complexidade assistencial.

Quanto aos pacientes de cuidados intensivos, para garantir a assistência adequada é necessário um número mínimo de profissionais, já que o paciente grave exige maior tempo de assistência na realização dos procedimentos em virtude das instabilidades clínicas. O sistema de classificação de pacientes, ao ser utilizado como uma ferramenta gerencial, permite estimar a quantidade de cuidado de enfermagem e planejar os recursos requeridos para atender as necessidades de assistência ao paciente em cada turno de trabalho. Nesse sentido, para prestar assistência com qualidade e segurança, torna-se imperativo adequar o número de trabalhadores de enfermagem à realidade de cada unidade.

Os dados encontrados refletem a sobrecarga de trabalho dos profissionais de enfermagem, comprometendo a qualidade dos serviços oferecidos, bem como na falta de segurança tanto para o paciente quanto para o profissional que a realiza.

0 estudo sobre o dimensionamento de pessoal de enfermagem e os resultados, permite às gerências tanto de enfermagem como da instituição conhecer melhor o perfil dos pacientes assistidos, o que facilita o provimento adequado de recursos humanos. Porém, como elementos que podem interferir e limitar os resultados do dimensionamento, e não foram avaliados totalmente neste estudo, temos as características da instituição tais como suas finalidades, objetivos, estrutura organizacional, planta física, tecnologias utilizadas e ainda a organização dos processos de trabalho.

Além disso, os achados dessa pesquisa possibilitam a abordagem e apresentam a necessidade de discussões sobre temas gerenciais que influenciam diretamente na assistência, como a internação de pacientes graves em unidades que não apresentam infraestrutura física e de recursos humanos para atender a todos os usuários adequadamente.

\section{REFERÊNCIAS}

1. Fugulin FMT, Gaidzinski RR, Castilho V. Dimensionamento de pessoal de enfermagem em instituições de saúde. In: Kurcgant P, organizadora. Gerenciamento em enfermagem. Rio de Janeiro: Guanabara Koogan; 2010. p. 121-35. 


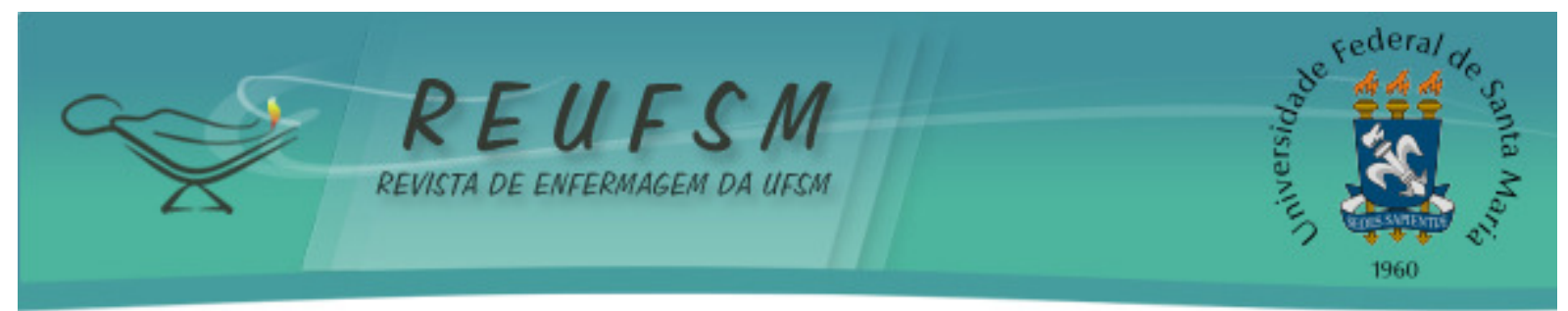

2. Cucolo DF, Perroca MG. Monitorando indicadores de desempenho relacionados ao tempo de assistência da equipe de enfermagem. Rev Esc Enferm USP. 2010;44(2):497-503.

3. Almeida ML, Segui MLH, Maftum MA, Labronici LM, Peres AM. Instrumentos gerenciais utilizados na tomada de decisão do enfermeiro no contexto hospitalar. Texto \& Contexto Enferm. 2011;20 (N Esp):131-7.

4. Fugulin FMT, Lima AFC, Castilho V, Bochembuzio L, Costa JA, Castro L, et al. Custo da adequação quantitativa de profissionais de enfermagem em Unidade Neonatal. Rev Esc Enferm USP. 2011;45 Esp:1582-8.

5. Rossetti AC. Carga de trabalho de profissionais de enfermagem em pronto socorro: proposta metodológica [dissertação]. São Paulo: Universidade de São Paulo, Escola de Enfermagem; 2010. 117 p.

6. Conselho Federal de Enfermagem. Resolução COFEN n 293, de 21 de setembro de 2004. Fixa e estabelece parâmetros para o dimensionamento do quadro de profissionais de enfermagem nas unidades assistenciais das instituições de saúde e assemelhados. [Internet]. 2004 [acesso em 2012 maio 5]. Disponível em: http://novo.portalcofen.gov.br/resoluo-cofen-2932004_4329.html.

7. Tsukamoto R. Tempo médio de cuidado ao paciente de alta dependência de enfermagem segundo o Nursing Activities Scores (NAS) [dissertação]. São Paulo: Universidade de São Paulo, Escola de Enfermagem; 2010. 108 p.

8. Zimmermann LP, Magnago TSBS, Urbanetto JS, Greco PBT, Viero NC, Vieira TG, et al. Avaliação do grau de dependência de cuidados de enfermagem dos pacientes internados em pronto socorro. Rev Enferm UFSM [Internet]. 2011 maio-ago [acesso em 2013 jun 6];1(2):153-63. Disponível em:

http://cascavel.ufsm.br/revistas/ojs-2.2.2/index.php/reufsm/article/view/2449/1659.

9. Araújo VB, Perroca MG, Jericó MC. Variabilidade do grau de complexidade assistencial do paciente em relação à equipe de enfermagem. Rev Latinoam Enferm. 2009;17(1):34-9.

10. Gil GP, Vituri DW, Haddad MCL, Vannuchi MTO, Moreno FN. Dimensionamento de pessoal de enfermagem e grau de dependência do paciente em um hospital universitário. Rev Eletrônica Enferm [Internet]. 2011 jul-set [acesso em 2013 jun 6];13(3):456-63. Disponível em: http://www.fen.ufg.br/fen_revista/v13/n3/pdf/v13n3a11.pdf.

11. Vituri DW, Lima SM, Kuwabara CCT, Gil RB, Évora YDM. Dimensionamento de enfermagem hospitalar: modelo OPAS/OMS. Texto \& Contexto Enferm. 2011;20(3):547-56.

12. Neis MEB, Gelbcke FL. Carga de trabalho na enfermagem: variável do dimensionamento de pessoal. Enferm Foco. 2011;2(1):6-9.

Data de recebimento: $24 / 06 / 2013$

Data de aceite: 07/11/2013

Contato com autor responsável: Anair Lazzari Nicola

Endereço postal: Sala 96, bloco de salas de aula da Unioeste Campus de Cascavel. Rua Universitária 2069, Jardim Universitário. CEP 85819-110. Cascavel - PR - Brasil.

E-mail: anairln@yahoo.com.br 